\title{
Strong decays of the higher isovector scalar mesons
}

\author{
Guan-Ying Wang, Shi-Chen Xue, Guan-Nan Li, En Wang, ${ }^{*}$ and De-Min $\mathrm{Li}^{\dagger}$ \\ Department of Physics, Zhengzhou University, Zhengzhou, Henan 450001, China
}

(Received 3 January 2018; published 27 February 2018)

\begin{abstract}
Under the assignment of $a_{0}(1450)$ as the ground isovector scalar meson, the strong decays of $a_{0}(1950)$ and $a_{0}(2020)$ are evaluated in the ${ }^{3} P_{0}$ model. Our calculations suggest that $a_{0}(1950)$ and $a_{0}(2020)$ can be regarded as the same resonance referring to $a_{0}\left(3^{3} P_{0}\right)$. The masses and strong decays of $a_{0}\left(2^{3} P_{0}\right)$ and $a_{0}\left(4^{3} P_{0}\right)$ are also predicted, which can be useful in the search for radially excited scalar mesons in the future.
\end{abstract}

DOI: 10.1103/PhysRevD.97.034030

\section{INTRODUCTION}

In the framework of quantum chromodynamics (QCD), apart from the ordinary $q \bar{q}$ states, other exotic states such as glueballs, hybrids, and tetraquarks are permitted to exist in meson spectra. To identify these exotic states, one needs to distinguish them from the background of ordinary $q \bar{q}$ states, which requires one to understand well the conventional $q \bar{q}$ meson spectroscopy both theoretically and experimentally.

Experimentally, $a_{0}(980), a_{1}(1260), b_{1}(1235)$, and $a_{2}(1320)$, respectively, as the lowest-lying $J^{P C}=0^{++}$, $1^{++}, 1^{+-}$, and $2^{++}$states, have been well established [1]. In contrast to $a_{1}(1260), b_{1}(1235)$, and $a_{2}(1320)$, which can be well accommodated in the ordinary $q \bar{q}$ picture, $a_{0}(980)$, together with its multiplet partners $\sigma, \kappa$, and $f_{0}(980)$, does not fit well into the predictions of the quark model. For example, the observed mass ordering of these lowest scalar states is $m_{\sigma}<m_{\kappa}<m_{a_{0}, f_{0}}$ [1], while in the conventional quark model, by a naive counting of the quark mass, the mass ordering of the scalar $q \bar{q}$ nonet should be $m_{\sigma} \sim m_{a_{0}}<m_{\kappa}<m_{f_{0}}$. These scalar states below $1 \mathrm{GeV}$ are generally believed not to be $q \bar{q}$ states [2-7].

At present, above the $a_{0}(980)$ mass, three higher isovector scalar states, $a_{0}(1450), a_{0}(2020)$, and $a_{0}(1950)$, have been reported experimentally. $a_{0}(1450)$ was observed in $p \bar{p}$ annihilation experiments [8,9], $D^{ \pm} \rightarrow K^{+} K^{-} \pi^{ \pm}$ [10], and $D^{0} \rightarrow K_{S}^{0} K^{ \pm} \pi^{\mp}$ [11]. $a_{0}$ (2020) with two alternative solutions of similar masses and widths was found by

\footnotetext{
*Corresponding author. wangen@zzu.edu.cn.

Corresponding author. lidm@zzu.edu.cn.

Published by the American Physical Society under the terms of the Creative Commons Attribution 4.0 International license. Further distribution of this work must maintain attribution to the author(s) and the published article's title, journal citation, and DOI. Funded by SCOAP ${ }^{3}$.
}

the Crystal Barrel Collaboration in the partial wave analysis of the data on $\bar{p} p \rightarrow \pi^{0} \eta$ and $\pi^{0} \eta^{\prime}$ [12], and $a_{0}(1950)$ was observed by the BABAR Collaboration in the processes $\gamma \gamma \rightarrow$ $K_{s}^{0} K^{ \pm} \pi^{\mp}$ and $\gamma \gamma \rightarrow K^{+} K^{-} \pi^{0}$ [13]. The masses and widths of the three isovector scalar states are listed in Table I. The lattice QCD calculations support that the lowest isovector scalar $q \bar{q}$ state corresponds to $a_{0}(1450)$ rather than $a_{0}(980)$ [14-16]. It is widely accepted that $a_{0}(1450)$ is the isovector member of the $1^{3} P_{0} q \bar{q}$ nonet [1]. The natures of $a_{0}(2020)$ and $a_{0}(1950)$ are unclear. To be able to understand the nature of a newly observed state, it is natural and necessary to exhaust the possible $q \bar{q}$ description before restoring to more exotic assignments. Therefore, with the assignment of $a_{0}(1450)$ as the ground $q \bar{q}$ state, one naturally asks whether the higher isovector scalar states, $a_{0}(2020)$ and $a_{0}(1950)$, can be identified as the radial excitations of $a_{0}(1450)$.

Theoretical efforts to identify the quark model assignments for $a_{0}(2020)$ and $a_{0}(1950)$ have been carried out. It is suggested that $a_{0}(1950) / a_{0}(2020)$ can be assigned as the $2^{3} P_{0}$ state based on the extended linear sigma model in Ref. [17], where $a_{0}(2020)$ is considered earlier evidence for $a_{0}(1950)$. In addition, $a_{0}(1950) / a_{0}(2020)$ is assigned as the $3^{3} P_{0}$ state based on the relativistic quark model in Ref. [18], where the predicted $a_{0}\left(3^{3} P_{0}\right)$ mass is about $1993 \mathrm{MeV}$, in agreement with both the $a_{0}(1950)$ and $a_{0}(2020)$ masses within errors. Obviously, further studies on the quark model assignments for $a_{0}(1950)$ and $a_{0}(2020)$

TABLE I. The masses and widths of the higher isovector scalar states (in MeV).

\begin{tabular}{lccc}
\hline \hline State & Mass & Width & Ref. \\
\hline$a_{0}(1450)$ & $1474 \pm 19$ & $265 \pm 13$ & {$[8,9]$} \\
$a_{0}(2020)$ & $2025 \pm 30$ & $330 \pm 75$ & {$[12]$} \\
$a_{0}(1950)$ & $1980_{-80}^{+12}$ & $225_{-32}^{+120}$ & {$[12]$} \\
\hline \hline
\end{tabular}


in other approaches are needed. Also, from Table I, one can see that the resonance parameters of $a_{0}(2020)$ are close to those of $a_{0}(1950)$. The observed mass difference between $a_{0}(1950)$ and $a_{0}(2020)$ is less than $100 \mathrm{MeV}$; in such a small mass interval, it would be very difficult to accommodate two radial excitations of $a_{0}(1450)$ in practically all the quark models. We therefore conclude that if both $a_{0}(2020)$ and $a_{0}(1950)$ can be explained as $q \bar{q}$ states, they should correspond to the same resonance. In this work, we shall discuss the possible quark model assignments of $a_{0}(2020)$ and $a_{0}(1950)$ by investigating their strong decays in the ${ }^{3} P_{0}$ model and check whether $a_{0}(2020)$ and $a_{0}(1950)$ can be identified as the same scalar meson.

The organization of this paper is as follows. In Sec. II, we give a brief review of the ${ }^{3} P_{0}$ model. In Sec. III, the calculations and discussion are presented, and the summary and conclusion are given in Sec. IV.

\section{THE ${ }^{3} P_{0}$ MODEL}

In this work, we employ the ${ }^{3} P_{0}$ model to evaluate the Okubo-Zweig-Iizuka (OZI)-allowed open flavor two-body strong decays of the initial meson. The ${ }^{3} P_{0}$ model, also known as the quark-pair creation model, was originally introduced by Micu [19] and further developed by Le Yaouanc et al. [20-22]. The ${ }^{3} P_{0}$ model has been widely applied to study strong decays of hadrons with considerable success [23-40]. The main assumption of the ${ }^{3} P_{0}$ model is that the strong decay occurs through a quark-antiquark pair with the vacuum quantum number. The new produced quark-antiquark pair, together with the $q \bar{q}$ within the initial meson, regroups into two outgoing mesons in all possible quark rearrangement ways.

Following the conventions in Ref. [31], the transition operator $T$ of the decay $A \rightarrow B C$ in the ${ }^{3} P_{0}$ model is given by

$$
\begin{aligned}
T= & -3 \gamma \sum_{m}\langle 1 m 1-m \mid 00\rangle \int d^{3} \boldsymbol{p}_{3} d^{3} \boldsymbol{p}_{4} \delta^{3}\left(\boldsymbol{p}_{3}+\boldsymbol{p}_{4}\right) \\
& \times \mathcal{Y}_{1}^{m}\left(\frac{\boldsymbol{p}_{3}-\boldsymbol{p}_{4}}{2}\right) \chi_{1-m}^{34} \phi_{0}^{34} \omega_{0}^{34} b_{3}^{\dagger}\left(\boldsymbol{p}_{3}\right) d_{4}^{\dagger}\left(\boldsymbol{p}_{4}\right),
\end{aligned}
$$

where $\gamma$ is a dimensionless parameter denoting the probability of the quark-antiquark pair $q_{3} \bar{q}_{4}$ with quantum number $J^{P C}=0^{++} \cdot \boldsymbol{p}_{3}$ and $\boldsymbol{p}_{4}$ are the momenta of the created quark $q_{3}$ and antiquark $\bar{q}_{4}$, respectively. $\chi_{1,-m}^{34}, \phi_{0}^{34}$, and $\omega_{0}^{34}$ are the spin, flavor, and color wave functions of $q_{3} \bar{q}_{4}$, respectively. The solid harmonic polynomial $\mathcal{Y}_{1}^{m}(\boldsymbol{p}) \equiv|p|^{1} Y_{1}^{m}\left(\theta_{p}, \phi_{p}\right)$ reflects the momentum-space distribution of the $q_{3} \overline{q_{4}}$.

The partial wave amplitude $\mathcal{M}^{L S}(\boldsymbol{P})$ of the decay $A \rightarrow$ $B C$ can be given by [41]

$$
\begin{aligned}
\mathcal{M}^{L S}(\boldsymbol{P})= & \sum_{\substack{M_{J_{B}}, M_{J_{C}} \\
M_{S}, M_{L}}}\left\langle L M_{L} S M_{S} \mid J_{A} M_{J_{A}}\right\rangle\left\langle J_{B} M_{J_{B}} J_{C} M_{J_{C}} \mid S M_{S}\right\rangle \\
& \times \int d \Omega Y_{L M_{L}}^{*} \mathcal{M}^{M_{J_{A}} M_{J_{B}} M_{J_{C}}}(\boldsymbol{P})
\end{aligned}
$$

where $\mathcal{M}^{M_{J_{A}} M_{J_{B}} M_{J_{C}}}(\boldsymbol{P})$ is the helicity amplitude and defined as

$$
\langle B C|T| A\rangle=\delta^{3}\left(\boldsymbol{P}_{A}-\boldsymbol{P}_{B}-\boldsymbol{P}_{C}\right) \mathcal{M}^{M_{J_{A}} M_{J_{B}} M_{J_{C}}}(\boldsymbol{P}) .
$$

$|A\rangle,|B\rangle$, and $|C\rangle$ denote the mock meson states defined in Ref. [42].

Due to different choices of the pair-production vertex, phase space convention, and employed meson space wave function, various ${ }^{3} P_{0}$ models exist in the literature. In this work, we employ the simplest vertex as introduced originally by Micu, who assumes a spatially constant pairproduction strength $\gamma$ [19], relativistic phase space, and simple harmonic oscillator (SHO) wave functions. With the relativistic phase space, the decay width $\Gamma(A \rightarrow B C)$ can be expressed in terms of the partial wave amplitude,

$$
\Gamma(A \rightarrow B C)=\frac{\pi|\boldsymbol{P}|}{4 M_{A}^{2}} \sum_{L S}\left|\mathcal{M}^{L S}(\boldsymbol{P})\right|^{2},
$$

where $|\boldsymbol{P}|=\frac{\sqrt{\left[M_{A}^{2}-\left(M_{B}+M_{C}\right)^{2}\right]\left[M_{A}^{2}-\left(M_{B}-M_{C}\right)^{2}\right]}}{2 M_{A}}$, and $M_{A}, M_{B}$, and $M_{C}$ are the masses of the mesons $A, B$, and $C$, respectively. The explicit expressions for $\mathcal{M}^{L S}(\boldsymbol{P})$ can be found in Refs. [31-33].

Under the SHO approximation, the meson space wave function in the momentum space is

$$
\psi_{n L M_{L}}(\boldsymbol{p})=R_{n L}^{\mathrm{SHO}}(p) Y_{L M_{L}}\left(\Omega_{p}\right),
$$

where the radial wave function is given by

$$
\begin{aligned}
R_{n L}^{\mathrm{SHO}}(p)= & \frac{(-1)^{n}(-i)^{L}}{\beta^{3 / 2}} \sqrt{\frac{2 n !}{\Gamma(n+L+3 / 2)}} \\
& \times\left(\frac{p}{\beta}\right)^{L} e^{-\left(p^{2} / 2 \beta^{2}\right)} L_{n}^{L+(1 / 2)}\left(\frac{p^{2}}{\beta^{2}}\right) .
\end{aligned}
$$

Here $\beta$ is the SHO wave function scale parameter, and $L_{n}^{L+(1 / 2)}\left(\frac{p^{2}}{\beta^{2}}\right)$ is an associated Laguerre polynomial.

\section{CALCULATION AND RESULTS}

In our calculations, the model parameters include the light nonstrange quark pair creation strength $\gamma$, the SHO wave function scale $\beta$, and the constituent quark masses. $\beta$ is set to be $\beta_{A}=\beta_{B}=\beta_{C}=0.4 \mathrm{GeV}$, the typical values used to evaluate the light meson decays, as in Refs. [25,26,31-35,39,43-45], and the constituent quark masses are taken to be $m_{u}=m_{d}=330 \mathrm{MeV}$ and $m_{s}=550 \mathrm{MeV}$, as in Refs. [27,31-35,39]. We take $\gamma=$ 7.1 by fitting to the total width of $a_{0}(1450)$ as the $1^{3} P_{0}$ state. The strange quark pair creation strength $\gamma_{s \bar{s}}$ can be related by $\gamma_{s \bar{s}}=\gamma \frac{m_{u}}{m_{s}}$ [46]. The meson flavor wave functions follow the conventions of Refs. [26,47] except for 
TABLE II. Decay widths of $a_{0}(1450)$ as the $1^{3} P_{0}$ state (in $\mathrm{MeV}$ ). The initial state mass is set to be $1474 \mathrm{MeV}$.

\begin{tabular}{lcr}
\hline \hline Channel & Mode & $\Gamma_{i}\left(1^{3} P_{0}\right)$ \\
\hline $0^{+} \rightarrow 0^{-} 0^{-}$ & $\pi \eta$ & 72.77 \\
& $\pi \eta^{\prime}$ & 144.47 \\
& $\pi \eta(1295)$ & 0.86 \\
& $K \bar{K}$ & 34.40 \\
$0^{+} \rightarrow 0^{-} 1^{+}$ & $\pi b_{1}(1235)$ & 9.96 \\
& $\pi f_{1}(1285)$ & 3.98 \\
Total width & & 266.45 \\
Experiment [1] & & $265 \pm 13$ \\
\hline \hline
\end{tabular}

$f_{1}(1285)=-0.28 n \bar{n}+0.96 s \bar{s}, \quad f_{1}(1420)=-0.96 n \bar{n}-$ $0.28 s \bar{s}$, as in Ref. [48], and $\eta(1295)=(n \bar{n}-s \bar{s}) / \sqrt{2}$, $\eta(1475)=(n \bar{n}+s \bar{s}) / \sqrt{2}$, as in Ref. [49], where $n \bar{n}=$ $(u \bar{u}+d \bar{d}) / \sqrt{2}$. Masses of the final state mesons are taken from [1].

The decay widths of $a_{0}(1450)$ as the $1^{3} P_{0}$ state are listed in Table II. The dominant decay modes of the $1^{3} P_{0}$ isovector state are $\pi \eta, \pi \eta^{\prime}$, and $K \bar{K}$, consistent with observations of $a_{0}(1450)$ [8,9,50].

The decay widths of $a_{0}(1950)$ as the $2^{3} P_{0}$ and $3^{3} P_{0}$ states are shown in Table III. If $a_{0}(1950)$ is the $2^{3} P_{0}$ state, its total width is expected to be about $771 \mathrm{MeV}$, much larger than the observed $a_{0}(1950)$ width of $271 \pm 22 \pm 29 \mathrm{MeV}$ [13]. The possibility of $a_{0}(1950)$ being the $2^{3} P_{0}$ state can be ruled out. If $a_{0}(1950)$ is the $3^{3} P_{0}$ state, its total width is about $207 \mathrm{MeV}$, reasonably close to the measurement within errors. The dependence of the total width of $a_{0}\left(3^{3} P_{0}\right)$ on the initial state mass is shown in Fig. 1.

TABLE III. Decay widths of $a_{0}(1950)$ as the $2^{3} P_{0}$ and $3^{3} P_{0}$ states (in MeV). The initial state mass is set to be $1931 \mathrm{MeV}$.

\begin{tabular}{lcrr}
\hline \hline Channel & Mode & $\Gamma_{i}\left(2^{3} P_{0}\right)$ & $\Gamma_{i}\left(3^{3} P_{0}\right)$ \\
\hline $0^{+} \rightarrow 0^{-} 0^{-}$ & $\pi \eta$ & 26.00 & 5.13 \\
& $\pi \eta^{\prime}$ & 2.07 & 2.52 \\
& $\pi(1300) \eta$ & 37.75 & 29.72 \\
& $\pi \eta(1475)$ & 20.81 & 13.22 \\
& $\pi \eta(1295)$ & 7.03 & 1.97 \\
& $K \bar{K}$ & 0.42 & 0.74 \\
$0^{+} \rightarrow 0^{-} 1^{+}$ & $\pi b_{1}(1235)$ & 339.48 & 90.06 \\
& $\pi f_{1}(1285)$ & 84.42 & 9.18 \\
& $\pi f_{1}(1420)$ & 4.38 & 0.61 \\
& $K K_{1}(1270)$ & 28.31 & 9.97 \\
& $K K_{1}(1400)$ & 2.38 & 0.72 \\
$0^{+} \rightarrow 1^{-} 1^{-}$ & $\eta a_{1}(1260)$ & 24.12 & 3.74 \\
& $\rho \omega$ & 181.18 & 36.07 \\
$0^{+} \rightarrow 0^{-} 2^{-}$ & $\pi \eta_{2}(1645)$ & 10.94 & 2.05 \\
Total width & & 1.24 & 1.58 \\
Experiment $[13]$ & & 770.51 & 207.27 \\
\hline \hline
\end{tabular}

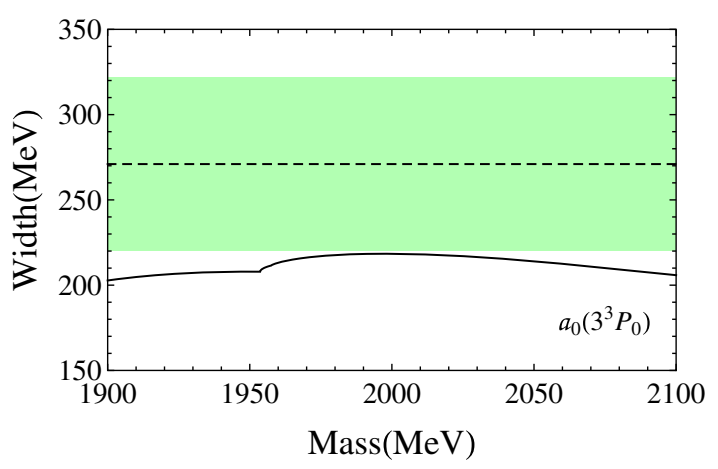

FIG. 1. The dependence of the total width of $a_{0}\left(3^{3} P_{0}\right)$ on the initial state mass. The dashed line with a green band denotes the $B A B A R$ experimental data [13].

Within the $a_{0}(1950)$ mass errors, the total width does not change too much. The assignment of $a_{0}(1950)$ as the $4^{3} P_{0}$ state can also be ruled out because the predicted width for $a_{0}\left(4^{3} P_{0}\right)$ with a mass of $1931 \mathrm{MeV}$ is about $37.3 \mathrm{MeV}$ (see also Fig. 3), much smaller than the $a_{0}(1950)$ width. Therefore, the measured mass and width for $a_{0}(1950)$ are in favor of it being the $3^{3} P_{0}$ state.

As shown in Fig. $1, a_{0}\left(3^{3} P_{0}\right)$ with a mass of $2025 \mathrm{MeV}$ is predicted to have a width of about $217 \mathrm{MeV}$, about $38 \mathrm{MeV}$ smaller than the lower limit of Crystal Barrel's solution I for the $a_{0}(2020)$ width of $330 \pm 75 \mathrm{MeV}$ [12], and the predicted width for $a_{0}\left(3^{3} P_{0}\right)$ with a mass of $1980 \mathrm{MeV}$ is about $218 \mathrm{MeV}$, in agreement with Crystal Barrel's solution II for the $a_{0}(2020)$ width of $225_{-32}^{+120} \mathrm{MeV}$ [12]. The possibility of $a_{0}(2020)$ being the $2^{3} P_{0}$ state can be ruled out because the expected width for $a_{0}\left(2^{3} P_{0}\right)$ with a mass of 1980 (2025) $\mathrm{MeV}$ is about 895 (995) MeV, much larger than the observed width of $a_{0}(2020)$, as shown in Table I. The predicted width for $a_{0}\left(4^{3} P_{0}\right)$ with a mass of 1980 (2025) $\mathrm{MeV}$ is about 36.8 (34.6) $\mathrm{MeV}$ (see also Fig. 3), much smaller than the $a_{0}(2020)$ width, which makes $a_{0}(2020)$ unlikely to be the $4^{3} P_{0}$ state. So, the

TABLE IV. Decay widths of $a_{0}\left(2^{3} P_{0}\right)$ (in $\mathrm{MeV}$ ). The initial state mass is set to be $1744 \mathrm{MeV}$.

\begin{tabular}{lcr}
\hline \hline Channel & Mode & $\Gamma_{i}\left(2^{3} P_{0}\right)$ \\
\hline $0^{+} \rightarrow 0^{-} 0^{-}$ & $\pi \eta$ & 7.91 \\
& $\pi \eta^{\prime}$ & 2.46 \\
& $\pi \eta(1475)$ & 19.25 \\
& $\pi \eta(1295)$ & 20.86 \\
$0^{+} \rightarrow 0^{-} 1^{+}$ & $K \bar{K}$ & 1.07 \\
& $\pi b_{1}(1235)$ & 213.08 \\
& $\pi f_{1}(1285)$ & 38.54 \\
$0^{+} \rightarrow 1^{-} 1^{-}$ & $\pi f_{1}(1420)$ & 1.02 \\
& $\rho \omega$ & 59.96 \\
& & 364.12 \\
\hline \hline
\end{tabular}




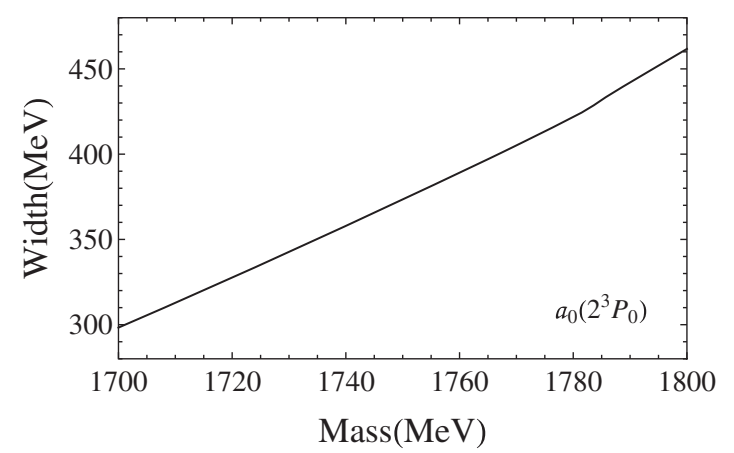

FIG. 2. The dependence of the total width of $a_{0}\left(2^{3} P_{0}\right)$ on the initial state mass.

measured mass and width for $a_{0}(2020)$ are consistent with an assignment of the $3^{3} P_{0}$ state.

The experimental evidence for both $a_{0}(1950)$ and $a_{0}(2020)$ turns out to be consistent with the presence of the same resonance corresponding to $a_{0}\left(3^{3} P_{0}\right)$. This naturally establishes $1.9 \mathrm{GeV}$ as the approximate mass for the $n \bar{n}$ members of the $3 P$ nonets, which could be useful to search for the $n \bar{n}$ members of the $3 P$ nonets experimentally. The dominant decay modes of $a_{0}\left(3^{3} P_{0}\right)$ are $\pi(1300) \eta, \pi \eta(1475), \pi b_{1}(1235), K K_{1}(1270)$, and $\rho \omega$.

$a_{1}(1640)$ and $a_{2}(1700)$ as the $2 P$ radial excitations have been established [25,51], which also fixes the natural mass scale for the $n \bar{n}$ members of the $2 P$ multiplets as about $1.7 \mathrm{GeV}$. One can expect to find $a_{0}\left(2^{3} P_{0}\right)$ near $1.7 \mathrm{GeV}$. At present, no candidate for the isovector scalar state around $1.7 \mathrm{GeV}$ is reported experimentally. An $a_{0}$-like pole associated to a resonance with a mass of about $1760 \mathrm{MeV}$ is found by investigating the meson-meson interaction in Refs. [52,53]. The $a_{0}\left(2^{3} P_{0}\right)$ mass in the extended linear sigma model is expected to be $1790 \pm 35 \mathrm{MeV}$ [17]. Systematic studies on the meson spectra in the relativistic quark models show that the expected $a_{0}\left(2^{3} P_{0}\right)$ mass is about $1679-1780 \mathrm{MeV}[18,47]$. Phenomenologically, it is suggested that the light mesons could be grouped into the following Regge trajectories [54],

$$
M_{n}^{2}=M_{0}^{2}+(n-1) \mu^{2},
$$

where $M_{0}$ is the lowest-lying meson mass, $n$ is the radial quantum number, and $\mu^{2}$ is the slope parameter of the corresponding trajectory. In the presence of $a_{0}(1450)$ and $a_{0}(1950) / a_{0}(2020)$ being the $1^{3} P_{0}$ and $3^{3} P_{0}$ states, respectively, the $a_{0}\left(2^{3} P_{0}\right)$ mass can be determined to be about $1744 \mathrm{MeV}$ based on Eq. (7), ${ }^{1}$ consistent with the extended linear sigma model prediction [17] and the quark model predictions $[18,47]$.

\footnotetext{
${ }^{1} \mathrm{We}$ take $\quad M_{a_{0}(1450)}=1474 \mathrm{MeV}, \quad M_{a_{0}(1950) / a_{0}(2020)}=$ $(1931+2025) / 2=1978 \mathrm{MeV}$, the average value of the $a_{0}(1950)$ mass reported by the $B A B A R$ Collaboration [13] and the favored solution for the $a_{0}(2020)$ mass [12].
}

TABLE V. Decay widths of $a_{0}\left(4^{3} P_{0}\right)$ (in $\mathrm{MeV}$ ). The initial state mass is set to be $2187 \mathrm{MeV}$.

\begin{tabular}{lcc}
\hline \hline Channel & Mode & $\Gamma_{i}\left(4^{3} P_{0}\right)$ \\
\hline $0^{+} \rightarrow 0^{-} 0^{-}$ & $\pi \eta$ & 0.00023 \\
& $\pi \eta^{\prime}$ & 0.41 \\
& $\pi(1300) \eta$ & 0.19 \\
& $\pi \eta(1475)$ & 0.77 \\
& $\pi \eta(1295)$ & 4.63 \\
& $K K(1460)$ & 0.37 \\
$0^{+} \rightarrow 0^{-} 1^{+}$ & $K b_{1}(1235)$ & 2.74 \\
& $\pi f_{1}(1285)$ & 0.0032 \\
& $\pi f_{1}(1420)$ & 0.09 \\
& $K K_{1}(1270)$ & 0.04 \\
& $K K_{1}(1400)$ & 4.27 \\
$0^{+} \rightarrow 1^{-} 1^{-}$ & $\eta a_{1}(1260)$ & 2.50 \\
& $\rho \omega$ & 0.34 \\
$0^{+} \rightarrow 0^{-} 2^{-}$ & $K^{*} \bar{K}^{*}$ & 4.26 \\
& $\pi \eta_{2}(1645)$ & 1.49 \\
\hline \hline
\end{tabular}

The strong decays of $a_{0}\left(2^{3} P_{0}\right)$ with a mass of $1744 \mathrm{MeV}$ are presented in Table IV. The total width of $a_{0}\left(2^{3} P_{0}\right)$ is expected to be about $364 \mathrm{MeV}$. The dominant decay modes of $a_{0}\left(2^{3} P_{0}\right)$ include $\pi \eta(1475), \pi \eta(1295), \pi b_{1}(1235)$, $\pi f_{1}(1285)$, and $\rho \omega$. The dependence of the total width of $a_{0}\left(2^{3} P_{0}\right)$ on the initial state mass is shown in Fig. 2. When the initial state mass varies from 1700 to $1800 \mathrm{MeV}$, the total width of $a_{0}\left(2^{3} P_{0}\right)$ varies from about 298 to $460 \mathrm{MeV}$. With the initial state mass of $1700 \mathrm{MeV}$, our predicted width of $298 \mathrm{MeV}$ is in agreement with the width of $293 \mathrm{MeV}$ expected by Ref. [25] for $a_{0}\left(2^{3} P_{0}\right)$.

$a_{0}\left(4^{3} P_{0}\right)$ is predicted to have a mass of about $2187 \mathrm{MeV}$ based on Eq. (7), consistent with $2250 \mathrm{MeV}$, the expected mass for $a_{0}\left(4^{3} P_{0}\right)$ in the quark model [18]. The strong decays of $a_{0}\left(4^{3} P_{0}\right)$ with a mass of $2187 \mathrm{MeV}$ are listed in Table V. The dependence of the total width of the $4^{3} P_{0}$ isovector state on the initial state mass is shown in Fig. 3. A

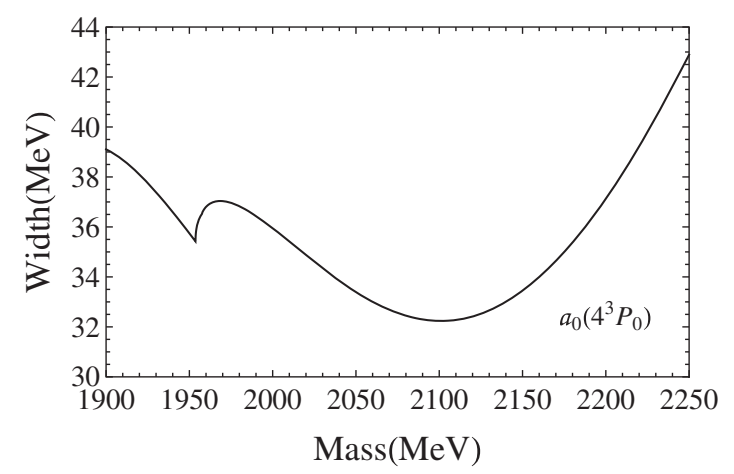

FIG. 3. The dependence of the total width of $a_{0}\left(4^{3} P_{0}\right)$ on the initial state mass. 
narrow width for $a_{0}\left(4^{3} P_{0}\right)$ is predicted. The $\pi \eta(1295)$, $K K_{1}(1270), \rho \omega$, and $\pi \eta_{2}(1645)$ channels are the dominant decay modes for $a_{0}\left(4^{3} P_{0}\right)$. As we can see in Figs. 1 and 3, the width derivatives are a discontinuity around $1950 \mathrm{MeV}$, which is because the decay channel $K K(1460)$ is open above this energy.

\section{SUMMARY AND CONCLUSION}

Observations of the state $a_{0}(1950)$ by the BABAR Collaboration have enlarged the family of the isovector scalar mesons. In this work, we discuss the possible quark model assignments of $a_{0}(1950)$ and $a_{0}(2020)$ by calculating their strong decays in the ${ }^{3} P_{0}$ model. We suggest that $a_{0}(1950)$ and $a_{0}(2020)$ can be regarded as the same resonance referring to $a_{0}\left(3^{3} P_{0}\right)$. The confirmation of $a_{0}(1950) / a_{0}(2020)$ as the $3^{3} P_{0}$ state thereby establishes about $1.9 \mathrm{GeV}$ as a natural mass scale for the $n \bar{n}$ members of the $3 P$ nonets.
In the presence of $a_{0}(1450)$ and $a_{0}(1950) / a_{0}(2020)$ being the $1^{3} P_{0}$ and $3^{3} P_{0}$ states, respectively, in Regge phenomenology, the masses of $a_{0}\left(2^{3} P_{0}\right)$ and $a_{0}\left(4^{3} P_{0}\right)$ are predicted to be about $1744 \mathrm{MeV}$ and $2187 \mathrm{MeV}$, respectively. The predicted masses for $a_{0}\left(2^{3} P_{0}\right)$ and $a_{0}\left(4^{3} P_{0}\right)$ are consistent with some other theoretical expectations. The total widths of $a_{0}\left(2^{3} P_{0}\right)$ and $a_{0}\left(4^{3} P_{0}\right)$ are expected to be about $364 \mathrm{MeV}$ and $36 \mathrm{MeV}$, respectively. Our predictions could be useful to study the higher isovector scalar mesons experimentally.

\section{ACKNOWLEDGMENTS}

This work is partly supported by the National Natural Science Foundation of China under Grants No. 11505158 and No. 11605158, the China Postdoctoral Science Foundation under Grant No. 2015M582197, the Postdoctoral Research Sponsorship in Henan Province under Grant No. 2015023, and the Academic Improvement Project of Zhengzhou University.
[1] C. Patrignani et al. (Particle Data Group), Review of particle physics, Chin. Phys. C 40, 100001 (2016).

[2] F. E. Close and N. A. Tornqvist, Scalar mesons above and below 1 GeV, J. Phys. G 28, R249 (2002).

[3] L. Maiani, F. Piccinini, A. D. Polosa, and V. Riquer, A New Look at Scalar Mesons, Phys. Rev. Lett. 93, 212002 (2004).

[4] C. Amsler and N. A. Tornqvist, Mesons beyond the naive quark model, Phys. Rep. 389, 61 (2004).

[5] J. R. Pelaez, On the Nature of Light Scalar Mesons from Their Large $N_{c}$ Behavior, Phys. Rev. Lett. 92, 102001 (2004).

[6] R. L. Jaffe, Exotica, Phys. Rep. 409, 1 (2005).

[7] G. Eichmann, C. S. Fischer, and W. Heupel, The light scalar mesons as tetraquarks, Phys. Lett. B 753, 282 (2016).

[8] C. Amsler et al. (Crystal Barrel Collaboration), Observation of a new $I^{G}\left(J^{P C}\right)=1^{-}\left(0^{++}\right)$resonance at $1450-\mathrm{MeV}$, Phys. Lett. B 333, 277 (1994).

[9] C. Amsler et al. (Crystal Barrel Collaboration), Coupled channel analysis of $\bar{p} p$ annihilation into $\pi^{0} \pi^{0} \pi^{0}, \pi^{0} \eta \eta$ and $\pi^{0} \pi^{0} \eta$, Phys. Lett. B 355, 425 (1995).

[10] P. Rubin et al. (CLEO Collaboration), Search for $C P$ violation in the Dalitz-plot analysis of $D^{ \pm} \rightarrow K^{+} K^{-} \pi^{ \pm}$, Phys. Rev. D 78, 072003 (2008).

[11] R. Aaij et al. (LHCb Collaboration), Studies of the resonance structure in $D^{0} \rightarrow K_{S}^{0} K^{ \pm} \pi^{\mp}$ decays, Phys. Rev. D 93, 052018 (2016).

[12] A. V. Anisovich et al. (Crystal Barrel Collaboration), $\bar{p} p \rightarrow$ $\pi^{0} \eta$ and $\pi^{0} \eta$ ' from $600 \mathrm{MeV} / \mathrm{c}$ to $1940 \mathrm{MeV} / \mathrm{c}$, Phys. Lett. B 452, 173 (1999).

[13] J. P. Lees et al. (BABAR Collaboration), Measurement of the $\mathrm{I}=1 / 2 K \pi \mathcal{S}$-wave amplitude from Dalitz plot analyses of $\eta_{c} \rightarrow K \bar{K} \pi$ in two-photon interactions, Phys. Rev. D 93, 012005 (2016).
[14] N. Mathur, A. Alexandru, Y. Chen, S. J. Dong, T. Draper, I. Horváth, F.X. Lee, K. F. Liu, S. Tamhankar, and J. B. Zhang, Scalar mesons $a_{0}(1450)$ and $\sigma(600)$ from lattice QCD, Phys. Rev. D 76, 114505 (2007).

[15] T. Burch, C. Gattringer, L. Y. Glozman, C. Hagen, C. B. Lang, and A. Schafer, Excited hadrons on the lattice: Mesons, Phys. Rev. D 73, 094505 (2006).

[16] S. Prelovsek, C. Dawson, T. Izubuchi, K. Orginos, and A. Soni, Scalar meson in dynamical and partially quenched two-flavor QCD: Lattice results and chiral loops, Phys. Rev. D 70, 094503 (2004).

[17] D. Parganlija and F. Giacosa, Excited scalar and pseudoscalar mesons in the extended linear sigma model, Eur. Phys. J. C 77, 450 (2017).

[18] D. Ebert, R. N. Faustov, and V. O. Galkin, Mass spectra and Regge trajectories of light mesons in the relativistic quark model, Phys. Rev. D 79, 114029 (2009).

[19] L. Micu, Decay rates of meson resonances in a quark model, Nucl. Phys. B10, 521 (1969).

[20] A. Le Yaouanc, L. Oliver, O. Pene, and J. C. Raynal, Naive quark pair creation model of strong interaction vertices, Phys. Rev. D 8, 2223 (1973).

[21] A. Le Yaouanc, L. Oliver, O. Pene, and J.-C. Raynal, Naive quark pair creation model and baryon decays, Phys. Rev. D 9, 1415 (1974).

[22] A. Le Yaouanc, L. Oliver, O. Pene, and J. C. Raynal, Hadron Transitions in the Quark Model (Gordon and Breach, New York, 1988).

[23] W. Roberts and B. Silvestre-Brac, General method of calculation of any hadronic decay in the $\mathrm{p}$ wave triplet model, Acta. Phys. Austriaca 11, 171 (1992). 
[24] H. G. Blundell, Meson properties in the quark model: A look at some outstanding problems, arXiv:hep-ph/9608473.

[25] T. Barnes, F. E. Close, P. R. Page, and E. S. Swanson, Higher quarkonia, Phys. Rev. D 55, 4157 (1997).

[26] T. Barnes, N. Black, and P. R. Page, Strong decays of strange quarkonia, Phys. Rev. D 68, 054014 (2003).

[27] F. E. Close and E. S. Swanson, Dynamics and decay of heavy-light hadrons, Phys. Rev. D 72, 094004 (2005).

[28] T. Barnes, S. Godfrey, and E. S. Swanson, Higher charmonia, Phys. Rev. D 72, 054026 (2005).

[29] B. Zhang, X. Liu, W. Z. Deng, and S. L. Zhu, $D_{s J}(2860)$ and $D_{s J}(2715)$, Eur. Phys. J. C 50, 617 (2007).

[30] G. J. Ding and M. L. Yan, $Y(2175)$ : Distinguish hybrid state from higher quarkonium, Phys. Lett. B 657, 49 (2007).

[31] D. M. Li and B. Ma, $X(1835)$ and $\eta(1760)$ observed by the BES Collaboration, Phys. Rev. D 77, 074004 (2008).

[32] D. M. Li and B. Ma, The $\eta(2225)$ observed by the BES Collaboration, Phys. Rev. D 77, 094021 (2008).

[33] D. M. Li and S. Zhou, Towards the assignment for the $4^{1} S_{0}$ meson nonet, Phys. Rev. D 78, 054013 (2008).

[34] D. M. Li and S. Zhou, Nature of the $\pi_{2}(1880)$, Phys. Rev. D 79, 014014 (2009).

[35] D. M. Li and E. Wang, Canonical interpretation of the $\eta_{2}(1870)$, Eur. Phys. J. C 63, 297 (2009).

[36] D. M. Li and B. Ma, Implication of BABAR's new data on the $D_{s 1}(2710)$ and $D_{s J}(2860)$, Phys. Rev. D 81, 014021 (2010).

[37] D. M. Li, P.F. Ji, and B. Ma, The newly observed open-charm states in quark model, Eur. Phys. J. C 71, 1582 (2011).

[38] Q.F. Lü and D. M. Li, Understanding the charmed states recently observed by the $\mathrm{LHCb}$ and BABAR Collaborations in the quark model, Phys. Rev. D 90, 054024 (2014).

[39] T. T. Pan, Q. F. Lü, E. Wang, and D. M. Li, Strong decays of the $X(2500)$ newly observed by the BESIII Collaboration, Phys. Rev. D 94, 054030 (2016).

[40] Q. F. Lü, T. T. Pan, Y. Y. Wang, E. Wang, and D. M. Li, Excited bottom and bottom-strange mesons in the quark model, Phys. Rev. D 94, 074012 (2016).
[41] M. Jacob and G. C. Wick, On the general theory of collisions for particles with spin, Ann. Phys. (N.Y.) 7, 404 (1959).

[42] C. Hayne and N. Isgur, Beyond the wave function at the origin: Some momentum dependent effects in the nonrelativistic quark model, Phys. Rev. D 25, 1944 (1982).

[43] H. G. Blundell and S. Godfrey, The $\Xi(2220)$ revisited: Strong decays of the $1^{3} F_{2}$ and $1^{3} F_{4} s \bar{s}$ mesons, Phys. Rev. D 53, 3700 (1996).

[44] H. G. Blundell, S. Godfrey, and B. Phelps, Properties of the strange axial mesons in the relativized quark model, Phys. Rev. D 53, 3712 (1996).

[45] E.S. Ackleh, T. Barnes, and E.S. Swanson, On the mechanism of open flavor strong decays, Phys. Rev. D 54, 6811 (1996).

[46] A. Le Yaouanc, L. Oliver, O. Pene, and J. C. Raynal, Why is $\psi^{\prime \prime}(4.414)$ so narrow?, Phys. Lett. 72B, 57 (1977).

[47] S. Godfrey and N. Isgur, Mesons in a relativized quark model with chromodynamics, Phys. Rev. D 32, 189 (1985).

[48] D. M. Li, H. Yu, and Q. X. Shen, Is $f_{1}(1420)$ the partner of $f_{1}(1285)$ in the ${ }^{3} P_{1} q \bar{q}$ nonet?, Chin. Phys. Lett. 17, 558 (2000).

[49] J. S. Yu, Z.F. Sun, X. Liu, and Q. Zhao, Categorizing resonances $\mathrm{X}(1835), \mathrm{X}(2120)$ and $\mathrm{X}(2370)$ in the pseudoscalar meson family, Phys. Rev. D 83, 114007 (2011).

[50] S. Uehara et al. (Belle Collaboration), High-statistics study of $\eta \pi^{0}$ production in two-photon collisions, Phys. Rev. D 80, 032001 (2009).

[51] P. R. Page, E. S. Swanson, and A. P. Szczepaniak, Hybrid meson decay phenomenology, Phys. Rev. D 59, 034016 (1999).

[52] C. Garcia-Recio, L. S. Geng, J. Nieves, and L. L. Salcedo, Low-lying even parity meson resonances and spin-flavor symmetry, Phys. Rev. D 83, 016007 (2011).

[53] L.S. Geng and E. Oset, Vector meson-vector meson interaction in a hidden gauge unitary approach, Phys. Rev. D 79, 074009 (2009).

[54] A. V. Anisovich, V. V. Anisovich, and A. V. Sarantsev, Systematics of $q \bar{q}$ states in the $\left(n, M^{2}\right)$ and $\left(J, M^{2}\right)$ planes, Phys. Rev. D 62, 051502 (2000). 